\title{
La encuesta, herramienta cognitiva ${ }^{1}$
}

\author{
Juan Javier Sánchez Carrión \\ Universidad Complutense de Madrid. Facultad de Ciencias Políticas y Sociología \\ jjsc@cps.ucm.es
}

Recibido: 10-03-2011

Aceptado: 26-04-2011

\begin{abstract}
Resumen
Este artículo analiza la simplificación que se produce al estudiar la encuesta en la perspectiva convencional y muestra la necesidad de considerarla desde una perspectiva cognitivoconstructivista. Ello implica entender que, como el iceberg, la encuesta está dividida en dos partes: una visible, constituida por los procedimientos técnico-estadísticos, y otra oculta, basada en los supuestos heurísticos y sociopolíticos sobre los que aquellos se sustentan. Dicho esto, se cuestionan los supuestos de base positivista —en lo metodológico- y liberal —en lo sociopolítico- que fundamentan el uso actual de la encuesta y se muestra la pertinencia de pensar en otros distintos, de naturaleza cognitivo-constructivista. A su vez, se introducen algunas consecuencias que tendría este cambio de supuestos sobre los procedimientos tal como ahora los conocemos. Por último, el artículo reflexiona sobre el tipo de sociedad en el que se enmarcan los supuestos actuales y el tipo de sociedad en el que se enmarcarían los alternativos.
\end{abstract}

Palabras clave: encuesta; sociología de la encuesta; constructivismo social.

\section{Abstract. Surveys, a cognitive tool}

This paper analyzes the simplification that occurs when studying surveys from a conventional perspective and demonstrates the need to study them from a of cognitive-constructivistperspective.. This implies seeing the surveys like an iceberg, divided in two parts: one visible, in the case of surveys constituted by technical and statistical procedures, and the other concealed, based on the heuristic and socio-political assumptions upon which the former are supported. Henceforth the paper questions the methodologically positivist and politically liberal assumptions upon which surveys are based and explains how pertinent it is to consider different assumptions of a cognitive and constructivist nature. At the same time, the paper introduces some of the consequences of these changes on the procedures

1. Este texto es una versión ampliada de otro que se publicó en el número 29 de la revista Inguriak (Sánchez Carrión, 2001a). Mi agradecimiento a José Manuel Segovia, que hizo el diseño de las figuras incluidas en este texto. 
as we know them now, changing conventional assumptions in the constructivist direction. Finally the paper considers the type of society that is framed by current assumptions and that which would be framed by the alternatives.

Key words: survey; sociology of surveys; social constructionism.

\section{Sumario}

1. Introducción

2. La idea del conocimiento como iceberg

3. Los supuestos de las herramientas cognitivas: heurísticos y sociopolíticos

4. Partes constitutivas de la encuesta y aplicación del modelo iceberg
5. Ejercicio de simulación: hacia un cambio en los supuestos heurísticos y sociopolíticos de la encuesta

6. Conclusión

Referencias bibliográficas

\section{Introducción}

Este trabajo tiene como objetivo presentar la encuesta como una herramienta cognitiva —-denominación alternativa a las de técnica o método-, que está compuesta de dos partes, a semejanza del iceberg: una parte visible, que tradicionalmente se identifica con la técnica (las fórmulas, los procedimientos, etc.), y otra oculta, consistente en los supuestos que sustentan a la primera y sin la cual carecería de sentido — no se mantendría operativa (figura 1).

La parte visible es la que tradicionalmente estudian los técnicos; la parte hundida la estudian los filósofos, los epistemólogos, los políticos, etc., sin que normalmente exista comunicación entre ambos grupos, en la medida que cada uno habla un lenguaje diferente — de hecho, suelen desconocer las materias del otro, más allá de unas ciertas nociones compartidas. Y el resultado de esta escisión es presentar la encuesta de una manera distorsionada, como si se tratara de un iceberg cuyas partes no encajan (figura 2).

Y los supuestos de los que hablamos en el primer párrafo son los propios de un tipo particular de sociedad, de la que dimana la propia encuesta, que son los de las sociedades occidentales surgidas a partir de la Ilustración. Sociedades diferentes favorecerían otros supuestos, sobre los cuales se construiría una encuesta (u otra herramienta cognitiva) también distinta.

A lo largo de estas páginas iré mostrando los supuestos que sustentan el uso de la encuesta y su aplicación a las diferentes partes que la componen, para concluir con un ejercicio de simulación que permita ver cómo les afecta a los procedimientos (la parte visible) el cambio de supuestos (la parte oculta) y, eventualmente, 1) cuál podría ser el tipo de herramienta cognitiva propio de unos supuestos distintos a los que sustentan el uso estándar de la encuesta, y 2) cómo habría de ser la sociedad que los hiciera, además de pensables cognitivamente, socialmente aceptables. Todo ello con la intención de mostrar las huellas que ha dejado la historia en una herramienta como la encuesta, que, en nuestro tiempo, pretende tener carta de naturaleza. 
Figura 1. La encuesta como herramienta cognitiva y el iceberg

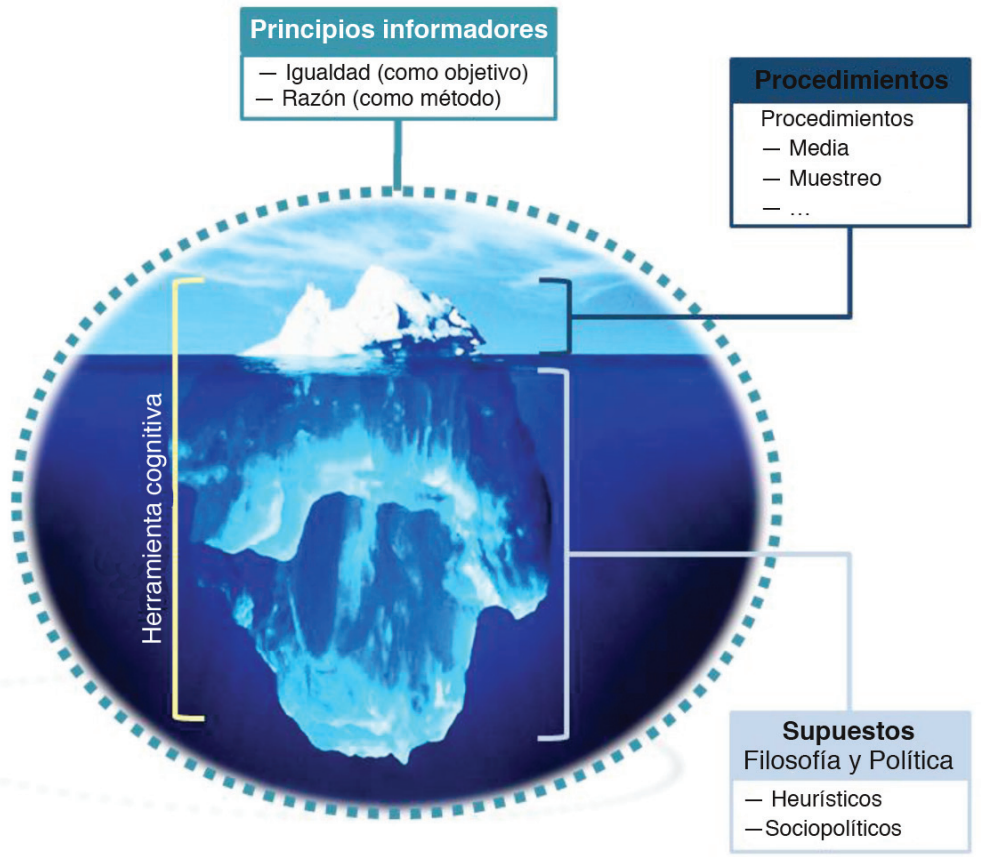

Figura 2. La encuesta como técnica y el iceberg

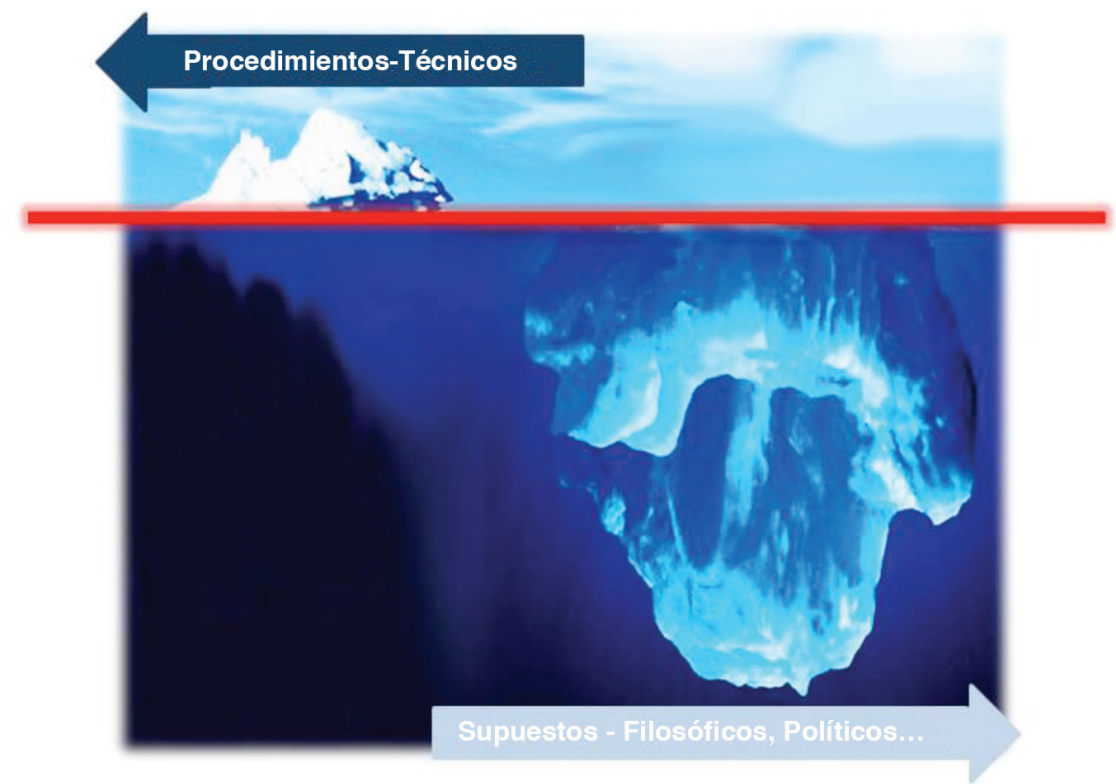




\section{La idea del conocimiento como iceberg}

Mi opinión es que lo que se hace (se ve) está en función de lo que se supone o se asume (no se ve). Un breve ejemplo aclaratorio espero que permita entender a qué me refiero. Resulta imposible usar la media aritmética, aunque se tenga conocimiento de las operaciones que están implicadas en su cálculo (sumar y dividir), si los sujetos a los que se les va a aplicar no son equivalentes y si no se ve la pertinencia de su utilización. Imaginemos el eventual uso de este sistema estadístico en la Inglaterra del siglo XVII — cuna de los aritméticos políticos, padres de la estadística-, en el contexto de la realización de una encuesta por un instituto que deseara conocer la opinión de los ingleses ante la restauración monárquica de Carlos II, tras la fase de gobierno de Cromwell.

En el caso de la encuesta que comentamos, ni los individuos eran iguales (equivalentes), separados como estaban en estamentos, ni a nadie le interesaba sus opiniones directas para legitimar la monarquía. Como resultado de estas dos circunstancias, malamente se podrían combinar sus opiniones para calcular una opinión media - como tendremos ocasión de ver más adelante, tampoco se podría sacar una muestra representativa de la población inglesa—, ni tendría sentido hacer una encuesta a la población para saber lo que pensaba — nadie estaba esperando su opinión para tomar decisiones.

En resumen, conocer las operaciones aritméticas que permiten calcular la media (parte visible del iceberg) no significa que se puedan aplicar al estudio de lo social —aunque, por ejemplo, sí a la astronomía para medir la posición verdadera de una estrella como la posición media de una serie de mediciones-, a menos que se cumplan ciertos supuestos (parte oculta), de los que tratamos a continuación. Es en este sentido en el que digo que lo que hacemos (lo que se ve) siempre está en función de lo que suponemos o asumimos (lo que no se ve).

\section{Los supuestos de las herramientas cognitivas: heurísticos y sociopolíticos}

Los supuestos de las herramientas cognitivas, como lo de todo aquello que se hace en nuestras sociedades, están relacionados con dos grandes principios informadores que tienen su origen en la Ilustración: la búsqueda de la igualdad, como objetivo, y el uso de la razón (la Ciencia sólo sería su máxima expresión), como método para acceder a la Verdad de las cosas — siendo la Verdad el referente de actuación en todos los campos de la vida. El primero de los principios es de naturaleza sociopolítica y el segundo, heurística, pero están completamente imbricados.

Estos dos grandes principios están detrás de los supuestos que subyacen al uso de la encuesta, también de naturalezas heurística y sociopolítica. Veamos cada uno de ellos, comenzando por los supuestos de tipo heurístico.

\subsection{Supuestos heurísticos}

Los supuestos heurísticos que sustentan el uso de la encuesta, basados en el positivismo (véase un repaso a las diferentes concepciones que existen sobre la 
ciencia, entre las que se incluye el positivismo, en Iranzo y Blanco, 1999), son de tres tipos, siguiendo la tipología establecida por Jesús Ibáñez (1992), y están relacionados entre sí en orden transitivo y descendente: el primero determina al segundo y éste, al tercero.

\section{Epistemológicos}

La pregunta asociada a este tipo de supuesto podría formularse en términos de ¿"para qué» hacemos la investigación? Y, al respecto, se pueden dar un par de respuestas: para reproducir (reflejar) o para construir lo observado (investigado). El que optemos por una u otra opción dependerá de la definición que hagamos previamente de cuáles son las naturalezas del objeto investigado, del sujeto que investiga y de cómo validamos el resultado de la investigación. En el caso de la encuesta, se parte de los supuestos de realismo del objeto y neutralismo del método, con lo que se buscará la reproducción del objeto observado como criterio para validar la calidad de la investigación, y, por tanto, ésta será la respuesta a la pregunta que nos formulábamos al principio de este párrafo.

\section{Metodológicos}

A este nivel, nos preguntamos ¿¿qué» hacer en la investigación? La respuesta dependerá de cuál sea la dimensión del objeto que estemos estudiando, que previamente, en el nivel inmediatamente superior (epistemológico), habremos definido como real o construido. A este respecto, siguiendo una tipología que en su momento estableciera Alfonso Ortí (1992), podemos hablar de diferentes dimensiones (niveles) de la «realidad», que son las que determinarán qué herramienta cognitiva utilizaremos para hacer la investigación. Así, podemos distinguir entre hechos y comportamientos (lo que se hace), en cuyo caso sería pertinente utilizar la herramienta estadística asociada a la realización de una encuesta; discursos (lo que se dice), lo que llevaría a la utilización de la herramienta lingüística, tal como se aplica en el grupo de discusión, y motivaciones (el porqué de lo que se hace), lo que llevaría al uso de la herramienta psicoanalítica y de la teoría de la racionalidad.

A este nivel, suponemos que la encuesta es la herramienta adecuada para estudiar la dimensión que hemos escogido del objeto de investigación.

\section{Tecnológicos}

En el nivel inferior, se encuentra la pregunta relacionada con el ¿¿cómo» hacemos la investigación?, y tiene que ver con la implementación de la herramienta seleccionada en el nivel anterior. Partiendo de los supuestos de realismo del objeto y neutralismo del método (nivel epistemológico), así como del hecho de que la encuesta es la herramienta adecuada para nuestra investigación (nivel metodológico), a nivel tecnológico se suele decir que, según cómo la hagamos (la encuesta), pueden aparecer errores derivados de lo que observamos (también llamados «errores de medición»), de lo que no observamos (también llamados «errores de muestreo») y del análisis que hagamos de lo observado. 
Los errores de medición se pueden atribuir al entrevistador, al entrevistado, al instrumento (el cuestionario) y/o al modo de generar la información (entrevista personal, telefónica, postal, en Internet, etc.). Los errores de muestreo suelen provenir de una deficiente cobertura del marco muestral utilizado para sacar la muestra, de la no respuesta de los entrevistados o, simplemente, del hecho de trabajar con una muestra, por muy bien que esté hecha. Por último, se cometen errores de análisis al grabar y procesar la información, además de al analizarla, quizá porque los datos incumplen los requisitos que exige la técnica utilizada (Cea d'Ancona, 2005; Groves, 1987, 1989; Sánchez Carrión, 1996).

A nivel tecnológico, vamos a hacer el supuesto de que no cometemos ninguno de los tres tipos de errores recién explicados. De que dichos supuestos técnicos se cumplan dependerá la «calidad» de la investigación —éste es el término que se utiliza en el contexto convencional de la encuesta para referirnos a lo bien o lo mal que esté hecha.

De manera resumida, podemos decir que el uso de la encuesta implica que hacemos los siguientes supuestos:

- En el nivel epistemológico: el objeto (aquello que queremos conocer: opiniones, hechos...) es real, la herramienta (la encuesta) es neutra y, por lo tanto, es posible reproducir lo investigado como forma de ver si la investigación está bien o mal hecha.

- En el nivel metodológico: la encuesta es la herramienta adecuada para conocer la dimensión sobre los hechos y los comportamientos del objeto investigado.

- En el nivel tecnológico: al hacer la encuesta, no cometemos errores de medida (en el nivel de lo observado), la muestra que sacamos es representativa de la población investigada (en el nivel de lo no observado) y la técnica de análisis utilizada se ajusta a los datos que tenemos (en el nivel del análisis).

\subsection{Supuestos sociopolíticos}

Si en el orden metodológico veíamos que el referente es el positivismo, en el orden sociopolítico el referente será el liberalismo político. También a este nivel vamos a distinguir entre tres supuestos distintos en los que se sustenta la encuesta. Veamos estos supuestos:

\section{Igualdad (formal) de los individuos}

En la encuesta, se supone que todos los individuos de la población investigada son (formalmente) iguales, desde el momento en que todos tienen el estatus de ciudadanos. Se trata de una extensión del modelo electoral aplicado a la encuesta, en la medida en que las elecciones son la base de legitimación de la actuación política y será ésta la que, utilizando los resultados obtenidos con dicha herramienta, permita resolver los problemas de los ciudadanos. Así como en las elecciones todo hombre, sin distinciones, vale un voto, también en la 
encuesta toda persona vale una opinión, sin que las de unas tengan más peso que las de otras, a semejanza del caso electoral.

La consecuencia práctica de este supuesto de igualdad es doble. Por un lado, permite (justifica) combinar opiniones - actitudes, comportamientos, etc. - de las personas para calcular medias. Por otro, da la posibilidad de intercambiar individuos de la población con el fin de diseñar muestras. A este respecto, cualquier individuo de la población vale para la muestra, con tal de que haya sido elegido al azar. Los elegidos están en representación del resto (los no elegidos); lo mismo que los elegidos en las elecciones - ahora por un procedimiento que no es de azar- representan al conjunto de la población. Cambia el método de elección, pero se mantiene la idea de representación.

\section{Bondad democrática de la encuesta}

El supuesto de que la encuesta es buena para la democracia está asociado a la idea de opinión pública, basada en la agregación de opiniones individuales e iguales, como expresión del sentir de una sociedad. La idea de opinión pública se va desarrollando desde finales del siglo XIX, con la figura pionera de Bryce (1889), hasta llegar al siglo XX, cuando cobra mayor importancia al quedar asociada al uso de las encuestas, que justamente se utilizan para poder medirla (véase Gallup y Rea, 1940). A partir de este momento, se supone que la encuesta es buena para la democracia porque:

1. Da la palabra a gente que, de otra manera, no sería escuchada por los gobernantes, por no tener la capacidad de formar lobbies.

2. Entre elecciones, permite al gobernante conocer (medir) la opinión de los gobernados, con lo que puede ajustar sus políticas contando siempre con lo que éstos piensan.

Llevado por su admiración hacia esta herramienta, Stouffer (1949) llegaría a decir que «las encuestas representan el instrumento más útil para la democracia que jamás se haya creado». Este supuesto de bondad democrática es básico para legitimar el uso de las encuestas en la sociedad y para hacer que las personas participen con sus respuestas.

\section{Existencia de sociedades integradas}

Suponemos que las sociedades donde se realizan las encuestas tienen un alto nivel de integración social, lo que justifica la participación en ellas de todos los individuos. Es decir, asumimos que, a priori, no hay razón para la no respuesta - lo mismo que se supone que tampoco la hay para la abstención electoral—y, por lo tanto, que es posible que se cumpla el segundo requisito que ha de satisfacer el muestreo para que sea representativo: además de la equiprobabilidad en la selección de los miembros de la muestra, la necesidad de que responda la gente seleccionada. Este supuesto es básico para considerar la no respuesta total en las encuestas como problema derivado tan sólo de la no disponibilidad (coyuntural) de los sujetos para contestar, lo cual facilita el desarrollo de proce- 
dimientos que tratan de resolverla a partir de esta consideración del problema (p.e. las sustituciones) y obvia así su importancia.

Como conclusión a todos los supuestos que acabamos de mencionar, tanto de naturalezas heurística como sociopolítica, diremos que, aunque todo el mundo entiende que no se pueden cumplir (al menos completamente y con seguridad), se mantienen como desiderátum por sus implicaciones positivas para el funcionamiento de la sociedad y para la realización de las encuestas, con lo cual se muestra el papel que desempeñan los intereses, además de las razones, en la conformación del conocimiento científico.

Soluciones «técnicas» eventuales a los problemas mencionados —obviando el cuestionamiento de los supuestos que subyacen a su utilización en la encuesta- suelen estar basadas en gastar más dinero, lo que quizá permita solucionar el problema en cuestión..., pero a costa de crear otros nuevos. Por ejemplo: para evitar la no respuesta, se puede pagar una cantidad importante de dinero a los entrevistados con el fin de conseguir su participación, lo que puede llevar a que, efectivamente, contesten..., pero ajustando sus respuestas a su nueva condición de «asalariados» del entrevistador; de esta manera, se evita un problema de falta de respuesta, pero a costa de crear otro de medición.

\section{Partes constitutivas de la encuesta y aplicación del modelo iceberg}

Veamos la aplicación de los supuestos anteriores, tanto heurísticos como sociopolíticos, a la encuesta, en función de sus diferentes partes constitutivas.

\subsection{El objetivo de la encuesta: explicar variabilidad}

Aplicando un instrumento de medida (un cuestionario) a una muestra sacada aleatoriamente de una población, obtenemos unos resultados que presentamos en forma de matriz de datos; es decir, en forma de tabla de doble entrada con las $p$ variables (preguntas) del estudio en columnas —en ningún caso aparecen constantes - y las $n$ unidades, desde la 1 hasta la $n$, en filas. A partir de esta matriz, que hay que recalcar que contiene solo variables, siguiendo la lógica del análisis explicativo, primero se mide, entre otras dimensiones, la variabilidad de la población (uso de la estadística descriptiva univariable), para pasar luego a explicarla (estadística bi-multivariable). El estudio será tanto más exitoso cuanta mayor parte de la variabilidad sea capaz de explicar (Sánchez Carrión, 1999a).

Ahora bien, para entender la originalidad de este planteamiento —es decir, para mostrar que no tiene nada de natural-, podemos hacernos la siguiente pregunta: ¿y por qué no explicar la homogeneidad —es decir, por qué hay gente que piensa, compra... lo mismo-, en lugar de la variabilidad observadas - es decir, por qué hay gente que compra, piensa... distinto? Se diría que, a priori, tanto explicar variabilidad como explicar homogeneidad tienen igual interés. Sin embargo, en la práctica, toda la investigación se organiza en torno a la explicación de la variabilidad. Pensemos en el caso de Coca-Cola o de un 
partido político que eventualmente realizaran una investigación por encuesta en un determinado país. En ambos casos, una vez constatado a partir de un análisis descriptivo que no todo el mundo consume o vota lo mismo, se trataría de explicar esa variabilidad (de consumo o de voto, respectivamente) para que, una vez explicada, es decir, una vez que se sabe a qué se debe, pueda ser controlada — tomando las medidas prácticas pertinentes- $y$, eventualmente, eliminada: lograr que todos consuman o voten lo mismo.

Es decir, la estadística explica la variabilidad, y los clientes, haciendo uso de este conocimiento, la controlan (reducen). El problema es que el día que, como consecuencia del éxito del procedimiento, no haya variabilidad (todos consuman lo mismo y todos voten al mismo partido), no habrá variables con las que construir la matriz de datos — solo habrá constantes- y, por lo tanto, tampoco se podrá utilizar la estadística para explicar esa hipotética situación de homogeneidad.

¿Seguro que no se podrá usar la estadística? Bueno, salvo que se dé un salto cualitativo y se cambien las unidades de análisis de la matriz de datos: de los individuos de nuestra primera matriz, ahora iguales (una vez que, hipotéticamente, se ha conquistado el mercado comercial y/o político del país donde se hizo el estudio), a los países, nuevas unidades de análisis de tipo agregado, todavía diferentes $y$, por lo tanto, susceptibles de ser investigadas estadísticamente - puesto que vuelve a haber variabilidad, ahora entre países. En términos prácticos, este cambio significaría pasar de la conquista del mercado nacional al internacional. $Y$, eventualmente, eliminada la variabilidad internacional -lo que ocurriría si todo el mundo mundial comprara o votara lo mismo-, salvo que encontremos otro ámbito de variabilidad que pueda ser estudiado (quizá la Luna u otro planeta), se acabó el trabajo del técnico estadístico. Podríamos decir, pues, que la estadística aplicada a la encuesta es una máquina formidable para crear entropía - reducir variabilidad-, que, en el límite, terminaría muriendo... de éxito. A esta capacidad la llamamos función entrópica de la estadistica.

En los párrafos anteriores, vemos de qué manera influye uno de los dos principios informadores de nuestras sociedades, el de la búsqueda de la igualdad, en la definición del objetivo de la investigación social mediante encuesta.

\subsection{La selección de la muestra}

Para contar una población numerosa, con el paso del tiempo se ha pasado del uso de multiplicadores (en los censos premodernos) a las monografías (tipo Le Play) y a las actuales encuestas por muestreo, tras la aceptación de las muestras por el Instituto Internacional de Estadística en $1926^{2}$. Y un requisito para poder hacer muestras, lo que explica el retraso de su aparición, es suponer la

2. Bajo el nombre de método representativo, el muestreo había sido presentado - y rechazado- - en 1895 a una reunión del mismo Instituto por Kaier, director de la Oficina Central de Estadística de Noruega. 
igualdad (al menos formal) de las personas que conforman la población objeto de estudio - algo impensable hasta tiempos históricos muy recientes. Esta igualdad de las personas es la que permite su intercambio para que unas (las seleccionadas) actúen en representación de otras (las no seleccionadas). Se trata de un proceso semejante al de la representación electoral, solo que modificando los procedimientos de selección: de la utilización del azar, en las muestras, a la selección intencional de las personas (los candidatos), en las elecciones.

Sólo la aceptación de la idea de representatividad política, tal como se expresa en los sistemas políticos actuales, permite la aceptación de la idea de representatividad estadística — siempre sobre la base de la igualdad (formal) de los individuos-, lo que hace posible que, cuando se publican resultados de encuestas en los que, a partir de una muestra, se sacan conclusiones para toda la población, los no entrevistados las acepten como si ellos hubieran participado (opinado). Es decir, se sientan representados por los que han tomado parte en la muestra. Lo mismo que ocurre en las elecciones, donde los no seleccionados para decidir (los representados) aceptan como si fueran propias las decisiones que adoptan sus representantes (sobre el muestreo Kish, 1965 -a un nivel avanzado-, y Kalton, 1983 - a un nivel intermedio—, son dos referencias obligadas).

\subsection{La medición}

A la hora de hacer encuestas, se asume que es posible ofrecer el mismo estímulo a todos los entrevistados, como base para aceptar como verdaderas las diferencias que se observan en sus respuestas. Complementario al supuesto anterior, que podríamos denominar de neutralidad del método, también se asume que existe un valor verdadero - supuesto de realismo del objeto - , que, justamente porque el método es neutro, es factible que sea medido sin alteración por parte del investigador. A partir de estos supuestos, se deriva toda la actuación de los investigadores, orientada a medir la fiabilidad y la validez de las mediciones hechas en la investigación social (Carmines y Zeller, 1979; Presser y otros, 2004a, 2004b; Saris y otros, 2004; Saris y Gallhofer, 2007).

Ahora bien, ¿es posible que todos los entrevistados reciban el mismo estímulo, independientemente de cuáles sean las condiciones que intervienen en la medición? Pensemos, a este respecto, en los efectos que tienen en las respuestas, tal como muestran múltiples investigaciones, variaciones en el instrumento (contenido y orden de las preguntas del cuestionario), en el entrevistador, en el entrevistado y en el modo de producir la información (encuestas postales, telefónicas, en la Web o con entrevistas personales), lo que hace que, dependiendo de las circunstancias de la encuesta (del estímulo), se obtengan distintos resultados al medir una misma «realidad» (Schuman y Presser, 1981; Converse y Presser, 1986; Fowler y Mangioni, 1990).

A modo de ejemplo, si preguntamos a la gente si está dispuesta a permitir la exhibición de películas X en televisión, el porcentaje de los que dicen que «no» es inferior al de los que responden que «sí» cuando la pregunta es si probibirían 
la exhibición de este mismo tipo de películas. Paradójico, si tenemos en cuenta que, en términos prácticos, no permitir algo es lo mismo que prohibirlo. Sin embargo, las respuestas difieren, lo cual muestra de qué manera al cambiar el estímulo cambia el resultado. Y el problema es que, en la práctica, solo podemos utilizar una forma (estímulo) para conocer la realidad, a sabiendas del efecto que tiene nuestra elección. ¿Cuál de ellas — si acaso lo es alguna- es la que permite conocer la verdadera opinión de los entrevistados?

En la literatura científica, el ejemplo del párrafo anterior, así como otros muchos que podríamos poner, se suele presentar mostrando la necesidad de hacer más investigación para superar los problemas — se suele decir que la disciplina es joven, por relación a otras ciencias-y llegar así a la forma correcta de medir la realidad (por ejemplo: utilizando la palabra prohibir en vez de permitir). Todo es cuestión de tiempo - $\mathrm{y}$ de dinero- para seguir haciendo más investigación, hasta que finalmente se llegue - porque seguro que se va a llegar - a la solución anhelada - piénsese en el uso generalizado de la expresión «todavía no se conoce...» en la investigación científica.

Sin embargo, con otra perspectiva de tipo constructivista, bien podrían atribuirse las diferencias obtenidas con distintos estímulos, sin pretensión de encontrar una solución al dilema, a la (in)oportunidad de asumir los supuestos de neutralismo del método y de realismo del objeto. Desde esta perspectiva, diríamos que cada forma de medir (por ejemplo: utilizando prohibir en lugar de permitir) arroja un resultado diferente, y todos son válidos, porque, desde esta perspectiva, no hay un valor verdadero que conocer ni (al no existir) la posibilidad de encontrar una forma neutra de medirlo.

\subsection{El análisis (descriptivo e inferencial)}

Con el paso del tiempo, el análisis ha ido evolucionando: desde el simple recuento de la población — saber cuánta gente hay y qué características tienehasta su modelización estadística - definir un orden subyacente a los datos obtenidos con el recuento. Y esta modelización primero sería univariable —el modelo más simple: la media - y luego multivariable — por ejemplo: la regresión como modelo más complejo. En todos los casos (recuento, media, regresión...), afloran los supuestos de los que estamos hablando en este trabajo, que, a medida que se fueron desarrollando y aceptando, hicieron posible los análisis estadísticos que hoy en día realizamos. Veamos el caso del recuento y de la modelización univariable (Desrosiéres, 1985, 1988, 1990, 1993, 1996; Porter, 1985; Sánchez Carrión, 1999b, 2000a, 2001a, 2001b, 2001c).

\section{El recuento}

A este respecto, hay que hablar de la dificultad histórica de contar y registrar a la población, tanto por su temor a ser contada, sabedora de que el recuento sólo iba a servir para pagar impuestos o para ir al ejército, como por los límites religiosos impuestos por la Iglesia al recuento civil de su pueblo. Por ejemplo: véase la Biblia y los casos de David — castigado por contar a su pueblo sin 
mandato Divino-y de Moisés — premiado por contar al pueblo de Israel, en este caso por orden de Dios (Torres Amat, 1894; Hecht, 1987). Es decir, para realizar algo tan simple como contar, lo primero que tienen que ganarse los contadores es la legitimidad necesaria para poder hacerlo. Y, de hecho, hasta el siglo XIX, no empezaron a generalizarse los registros civiles y la realización de censos, a medida que el estado iba introduciéndose en la vida de las personas ofreciéndoles servicios y no solo haciendo la guerra.

En este desarrollo del recuento civil, tuvo importancia el proceso de individualización occidental, que permitió pasar del recuento de vecinos o fuegos, unidades agregadas propias de los censos realizados hasta el siglo XVIII, al de habitantes, que es la unidad utilizada en la actualidad. También lo tuvo el cambio de políticas de las naciones (del mercantilismo a la fisiocracia y luego al laissez-faire...) para explicar cómo cambiaron los objetivos que se atribuía a los censos y cómo esto se fue reflejando en sus mismas preguntas.

Y no menos importante que los factores anteriores fue el papel de la ciencia en el proceso de legitimación del recuento de la población, al presentarlo como método y fuente de una manera distinta de argumentar, no escolástica, basado en la utilización, como decía Petty (1676), padre de la aritmética política, de número, peso y medida, en lugar de «utilizar solo palabras comparativas y superlativas, y argumentos intelectuales». Este respaldo de la ciencia sería básico para hacer atractivo el recuento a los ojos de los contados, y así lo vieron los administradores que buscaron el apoyo de los científicos - como antaño buscaron el de la Iglesia - para que les facilitasen el trabajo — científicos que, a su vez, se beneficiaron del papel preferente que les otorgaba la Administración, en un claro proceso simbiótico.

\section{La modelización estadistica univariable}

Una vez que fue posible contar, se empezaron a observar regularidades. De hecho, el propio Graunt, cofundador con el mencionado Petty de la aritmética política, es un pionero en la constatación de regularidades en el campo de los fenómenos demográficos, incluido el suicidio (Graunt, 1661). Los contadores observaron que, año tras año, se repetía el mismo (o parecido) número de suicidios, de matrimonios, de fallecimientos, de delitos..., para lo cual tenían que buscar una explicación. Y pasado un primer momento en el que todavía se buscó la explicación en la voluntad divina, la ciencia (estadística) iría haciendo su aparición para proporcionar una explicación alternativa ${ }^{3}$.

Y las explicaciones que se dan al recuento, tal como tratamos de mostrar en este artículo al presentar la encuesta como una herramienta cognitiva, dependerán de los supuestos de partida de los investigadores. El caso de la media,

3. Ejemplo de esa forma religiosa de buscar explicaciones es la que Arbuthnott (1710) da, en el siglo XVII, al número constante de nacimientos anuales, con igual número de mujeres que de hombres: «[la Divina Providencia] garantiza que las Especies nunca falten ni perezcan, puesto que cada Macho puede tener su Hembra, y de una edad proporcional. Esta Igualdad de Machos y de Hembras no es producto del Azar, sino de la Divina Providencia trabajando para un buen fin, que a continuación demuestro». 
la desviación típica y la distribución normal, así como su aplicación según Quetelet, Galton y Pearson al estudio de lo social, serán los ejemplos que comentaremos para ilustrar lo que decimos.

El precursor más importante de la aplicación de la media a lo social fue Quetelet $(1835,1869)$. En combinación con la distribución normal, la media servía para modelizar las regularidades observadas en la altura de los soldados, que era el ejemplo por él utilizado. Según decía el autor, si se nos pregunta cuál será la altura de una persona, no podremos dar una respuesta cierta. Ahora bien, podremos saber cuál será la altura media de la población y qué porcentaje de personas encontraremos en cualquier intervalo de su distribución. Y ello es así porque existen leyes de tipo social, semejantes a las de la naturaleza, a las que están sometidos los fenómenos sociales (morales, diría el autor), y que son las que explican las regularidades observadas - en este caso, esa altura media. Y, además, y ésta es la contribución original de Quetelet, esas leyes sociales se pueden expresar en forma estadística, en concreto, mediante el uso de la media de una distribución normal, de la cual conocemos sus propiedades. Y como resultado de la existencia de esas leyes, está el hombre medio, concepto que Quetelet desarrolla para referirse a la tendencia (social) que se da, producto de causas constantes, a que los individuos se aproximen a un valor promedio. Causas variables y accidentales son las que explicarían que no todos los individuos tengan ese valor — caso de características cuantificables, como la altura- o que no tengan el mismo comportamiento — caso del suicidio.

En su idea de hombre medio, no cabía incluir la desviación típica, puesto que las desviaciones respecto a la media eran errores, producto, como decíamos en el párrafo anterior, de causas variables y accidentales; por eso el autor no le prestó atención a este valor estadístico. Sí se la prestó a la distribución normal, porque, fuera de este tipo de distribución, la media carecía de sentido - a diferencia de lo que ocurre hoy en día, en que la media aritmética, medida de tendencia (ahora central), se utiliza profusamente sin considerar el tipo de distribución a la que pertenece.

Tuvieron que pasar algunos años antes de que el hombre medio de Quetelet, modelo estático de todo lo bueno y lo bello, terminara por ser considerado un hombre mediocre, como corresponde a alguien que tiene los valores medios en una sociedad en la que Darwin acaba de decir que las especies evolucionan (el modelo de orden social se hace dinámico) y que solo los más fuertes sobreviven (con la lucha por la supervivencia, frente a la selección natural, como motor de cambio). En estas circunstancias, ¿cómo ignorar la desviación típica, cuando, como decimos, la variabilidad - la diferencia entre individuos- es el nuevo motor de la evolución? Así fue como Galton (1889), primo del famoso científico evolucionista, primero construyó el recorrido intercuartílico y posteriormente la desviación típica, como medidas de variabilidad.

¿Y la forma de la distribución, por completar la tríada de las medidas de resumen univariable que se suelen utilizar en estadística? Justamente la idea, derivada de la observación de alguna distribución estadística (la altura de los 
soldados), de que las distribuciones de las características de los individuos (no solo físicas, sino también morales) tienen forma normal es lo que lleva a Quetelet a justificar su idea de hombre medio. Y son las variaciones por relación a este hombre medio los errores mínimos de las colas de la distribución normal. Por tanto, para Quetelet solo existen curvas normales, que son las que legitiman su modelo social (estadístico) de hombre medio. En el caso de Pearson (1892), darwinista de pro relacionado con Galton, aceptar la idea de que las distribuciones de las características de las personas tienen una forma normal sería aceptar la idea de que existe una tendencia a la normalidad (a la mediocridad, a los valores medios), que justo es contraria a su tesis de la supervivencia de los más fuertes, lo que le llevaría a no interesarse por la distribución normal y a desarrollar las distribuciones asimétricas.

Reflexiones semejantes a las del párrafo anterior se podrían hacer sobre otros valores estadísticos, univariables y multivariables, y todas coincidirían en mostrar la importancia de los supuestos a la hora de entender su utilización. Por ejemplo: desde el punto de vista de la estadística inferencial, es necesario entender la figura de Pascal, quien, a partir del siglo XVII, inicia un proceso de intento de control de la incertidumbre (la suerte) en torno a los juegos de azar - conocer no ya lo que existe (lo observado), sino lo que no se ha producido todavía (lo no observado) — , papel hasta entonces reservado a la figura de Dios, mediante el uso de la ciencia (el cálculo de probabilidades) (Coumet, 2000).

En términos inferenciales, el supuesto heurístico de que existe un valor verdadero es el que permite que, a la hora de hacer estimaciones de parámetros poblacionales, podamos utilizar los conceptos de sesgo y error variable. El sesgo o error fijo de muestreo implica la existencia de un valor verdadero (el parámetro poblacional) por relación al cual medimos su magnitud ${ }^{4}$. En el caso del error variable, también se hace necesario asumir la existencia de un valor verdadero (igualmente, el parámetro poblacional), y por tanto único, en torno al cual fluctúan las infinitas e hipotéticas muestras que pudiéramos sacar de la población. Cuanto mayor sea la fluctuación, mayor será el error. Es decir, toda la estadística inferencial gira sobre la existencia de un valor que asumimos como cierto, aunque en la práctica sea desconocido — isi lo conociéramos, para qué íbamos a hacer la encuesta!

\section{Ejercicio de simulación: hacia un cambio en los supuestos heurísticos y sociopolíticos de la encuesta}

En este apartado, vamos a pensar cómo afectaría a la encuesta (a la estadística) la existencia de otros supuestos distintos a los que están detrás de su uso convencional. Veamos los cambios al nivel de los supuestos (heurísticos y sociopolíticos) y sus implicaciones al nivel de los procedimientos. Por último,

4. El sesgo es igual a la diferencia entre el valor esperado de la variable estadística y el parámetro poblacional. 
puesto que no puede haber ciencia nueva en una sociedad vieja, pensemos cómo sería la sociedad que sustentara esos nuevos supuestos: ¿cuáles serían sus principios informadores?

\subsection{Cambios en el ámbito de los supuestos}

\subsubsection{Supuestos heurísticos}

$\mathrm{Si}$, en el marco de la visión convencional de la encuesta, el referente metodológico es el positivismo, en el de esta perspectiva cognitiva lo será el constructivismo 5 . Como dijéramos al principio del artículo, los supuestos heurísticos son de tres tipos: epistemológicos, metodológicos y tecnológicos. Veamos cada uno de ellos, pero ahora en esta nueva perspectiva.

\section{Epistemológicos}

Del «para qué» igual a reproducir la realidad, tal como se asume en la perspectiva convencional de la encuesta, al "para qué» igual a construirla, como alternativa constructivista. Frente a la epistemología realista (positivista) que subyace al uso de la encuesta, cabe pensar en el uso de una epistemología constructivista que asuma el no realismo del objeto y la no neutralidad del método, así como que parta de la identidad parcial entre el objeto (el entrevistado) y el sujeto (el entrevistador) de la investigación — desde papeles diferentes (sociales pero no epistemológicos) en el momento de la entrevista, ambos se investigan. Producto de esta identidad, podemos decir que, frente a la reproducción fiel de lo observado (la realidad), que es el criterio utilizado para ver la calidad de un conocimiento en la lógica realista, dentro de una visión constructivista habrá que proponer la praxis como criterio de validación de la investigación: el saber será bueno (que no verdadero) si es operativo, o, dicho en otros términos, si «funciona».

\section{Metodológicos}

Desde el momento en que se rechaza la idea de verdad (o realidad independiente del investigador), se difumina la distinción que se establece entre lo que se hace (hechos o comportamientos, supuestamente susceptibles de ser conocidos objetivamente) y lo que se dice (discursos, opiniones... no susceptibles de ser conocidos objetivamente), que, junto a las motivaciones (por qué se hace o se dice), son las dimensiones susceptibles de ser estudiadas en los objetos. Y si se difumina la distinción entre las dimensiones de los objetos, ello implica que se difumina la distinción entre las herramientas con las que se investigan.

5. Se puede ver un repaso de las diferentes concepciones postpositivistas de la ciencia, todas con el común denominador de incluir lo social como parte constitutiva de la producción de conocimiento científico, en Iranzo y otros (1995) e Iranzo y Blanco (1999). En el ámbito de la encuesta, autores como Cicourel (1964) y, más recientemente, Bourdieu (2001) son buenas referencias de posiciones críticas con el enfoque convencional de esta herramienta, en línea con la argumentación seguida en nuestro artículo. 


\section{Tecnológicos}

El cambio fundamental a este nivel es que, en vez de hablar de errores cometidos en la implementación de la encuesta (de no observación, de mala observación o de mal análisis de lo observado), que en la perspectiva convencional asumimos que no se producen, tendríamos que hablar de efectos, en la medida que, en la nueva perspectiva, no hay un valor verdadero en relación al cual comparar lo correcto de la medición. Si mediciones diferentes (hechas con distintas formulaciones de una pregunta, el uso de distintos modos de generar la información...) dan resultados distintos, y no hay uno verdadero, tendremos que hablar del efecto que tiene cada forma de medir y no del error que se comete con la misma.

Por lo demás, en esta perspectiva habrá que sustituir el concepto «calidad» por el de «bondad» de la encuesta, de connotaciones técnicas el primero y cognitivas el segundo.

\subsubsection{Supuestos sociopolíticos}

$\mathrm{Si}$, en el orden metodológico, hemos dicho que el referente de estos nuevos supuestos es el constructivismo, en el sociopolítico el referente está por definir. En relación con la encuesta como herramienta cognitiva también en el nivel de los supuestos sociopolíticos vamos a hablar de tres supuestos, tal como se muestra a continuación.

\section{(Des)igualdad (material) de los individuos}

Frente a la igualdad (formal) de los individuos, que es lo que se asume en la investigación convencional, vamos a asumir su desigualdad (material). Se trata de un supuesto, este último, que funciona en la investigación comercial, donde no se asume que todos los individuos sean iguales, sino que el valor de cada persona está en función de su capacidad de ser cliente del que investiga, pero no en la social, en la que, como digo, todos somos iguales - igual supuesto que el que regula los procesos electorales. Es decir, la idea de un hombre un voto, que es la que impera en el campo de lo sociopolítico, en el comercial se transforma en un euro un voto.

En la medida que el supuesto de igualdad está relacionado con el papel de las elecciones como único proceso legitimado para acceder al poder político, que a su vez es el que puede (debe) resolver los problemas de los ciudadanos, si se quiere cuestionar la pertinencia de este supuesto, será necesario relativizar el papel de los procesos electorales en la construcción de una sociedad democrática, introduciendo otros elementos (reconociendo los existentes) de participación social y política que complementen la función que ahora tienen, en exclusiva, las elecciones.

\section{(Seudo)bondad democrática de la encuesta}

Del supuesto de desigualdad material de los individuos, se deriva una crítica a la idea de opinión pública («ficción del estado de derecho», según Habermas, 2004, u «obsesión de la conciencia democrática», según Derrida, 1992) y, por 
lo tanto, a la encuesta como herramienta con la que medirla. A este respecto, es de interés la crítica de Bourdieu $(1973,1984)$ a los sondeos de opinión y a sus supuestos. Según el autor, contrariamente a lo que se asume, cabe tener en cuenta que:

a) No todo el mundo tiene una opinión acerca de todo lo que se investiga.

b) No todas las opiniones tienen el mismo peso y uel hecho de acumular opiniones que no tienen en absoluto la misma fuerza real lleva a producir artefactos desprovistos de sentido».

c) No hay consenso en los problemas que hay que investigar, como parece desprenderse de las preguntas de las encuestas.

La tercera parte implica que, contrariamente a lo que se suele opinar, en el sentido de que las encuestas sirven para extraer información de la sociedad, también introducen información en la misma. Ello es así porque al preguntar, por ejemplo, sobre los problemas que la gente considera más importantes, elegidos de una lista leída por el entrevistador, la misma lista está mostrando como importantes unos (los que considera el investigador) y dejando aparte otros (no considerados por el investigador, pero que a lo mejor son importantes para los entrevistados).

En términos pragmáticos, quizá la crítica segunda sea la más importante. Y no solo la formula Bourdieu, sino que también Blumer (1948), en su presentación a la Asamblea Anual de la Sociedad Norteamericana de Sociología, celebrada en 1947, criticaba el hecho de que el muestreo probabilístico no tuviera en cuenta la estructura de las sociedades modernas, donde existen relaciones de poder e influencia, intereses particulares y grupos de presión. Es por todo ello que no todas las opiniones ni todos los que emiten opiniones tienen el mismo peso ni la misma capacidad de influir en los acontecimientos y en las decisiones políticas.

\section{Existencia de sociedades (des) integradas}

En este ejercicio de simulación, se supone la existencia de sociedades conflictivas que no permiten asumir la participación de todos los individuos ni en las encuestas ni en las elecciones. Y, por lo tanto, en vez de preguntarnos por qué hay gente que no participa en las encuestas (también en las elecciones), habrá que preguntarse por qué hay gente que sí lo hace. Una consecuencia de este supuesto es que hay que admitir que la no respuesta (sea electoral o estadística) es consustancial a nuestras sociedades $\mathrm{y}$, por ello, no se puede evitar. Por lo tanto, es necesario prestar una consideración especial al colectivo de no respondientes, empezando por preguntarse si está justificado sacar conclusiones solo a partir de los que contestan, considerando a los que no lo hacen solo como no disponibles — su única diferencia con los respondientes.

De hecho, cuando en las encuestas se utilizan las sustituciones como forma de tratar el problema de la no respuesta, estamos asumiendo la no dispo- 
nibilidad de los entrevistados como explicación de la no respuesta ${ }^{6}$. Para la encuesta, sustitutos y sustituidos son iguales, con la única diferencia de que los primeros están disponibles para realizarla y los segundos, no. También se hace el supuesto de no disponibilidad - y de aquí el origen de la primera confusión - cuando, a la hora de definir los requisitos para conseguir una muestra representativa, tal como aparece en los libros de estadística, se hace referencia exclusivamente a la forma aleatoria de seleccionar a sus miembros, pero sin consideración alguna al hecho de que, una vez seleccionados, han de contestar.

\subsection{Cambios en el nivel de la técnica (herramienta cognitiva)}

\subsubsection{El objetivo de la encuesta: explicar homogeneidad}

A la hora de hacer el análisis de los datos, se trataría de explicar homogeneidad en lugar de variabilidad, que es lo que se hace en la perspectiva convencional. Las preguntas que tendríamos que formularnos serían del tipo de las que siguen: ¿qué tiene de problemática la variabilidad (ser diferente), para que justifique la necesidad de su estudio? ¿Acaso la variabilidad sería intrínsecamente más interesante que la homogeneidad si no fuera por la necesidad (retórica) de «suprimir las desigualdades»? ¿Cómo sería una estadística preocupada por la homogeneidad y que, por tanto, intentara explicarla? ¿Sería posible una estadística con otros valores no homogeneizadores, tal como en su momento intentó Tukey (1977), al presentar su Análisis exploratorio de los datos como alternativa a las medidas clásicas de descripción de una variable (tres, igual número que las medidas que permiten clasificar a las mises de los concursos de belleza: tendencia, dispersión y forma)?

\subsubsection{La selección de la muestra}

$\mathrm{Si}$ los individuos no son iguales, tampoco son intercambiables; y si no son intercambiables, ¿cómo conseguir que la muestra sea representativa sin que estén incluidos todos los miembros de la población? En esta nueva situación, ¿aceptarían los resultados de la encuesta las personas no seleccionadas en la muestra? Pensemos en el caso de las muestras comerciales, donde no todo el mundo tiene la misma (algunas personas ni siquiera tienen) probabilidad de ser elegido(as), puesto que la probabilidad de cada miembro de la población está en función del peso que tiene para el problema investigado. Pensemos

6. Distinto es el caso de aquellas encuestas que, en lugar de las sustituciones, realizan re-visitas (nuevos intentos de contacto), con el fin de lograr entrevistar a las unidades de muestreo seleccionadas y que están ausentes en el momento de hacer las preguntas. Mientras que, para los investigadores que sustituyen, dado que siempre es posible encontrar un sustituto, el tema de la no respuesta tiene escasa importancia, en el caso de los investigadores que no admiten las sustituciones existe una gran preocupación por la no respuesta, pues se entiende que puede comprometer la representatividad de las muestras. Su actuación se dirige, primero, a minimizarla, y luego, cuando no se puede evitar, a controlar el efecto que pueda tener (Díaz de Rada, 2000; Goyder, 1987; Groves y Cooper, 1998; Sánchez Carrión, 2000b; Stoop, 2006; Stoop y otros, 2010). 
también en el caso de la realización de encuestas en países de escasa tradición democrática, donde los resultados obtenidos con muestras no son aceptados por las personas que no forman parte de la misma - no aceptan su representatividad-, lo cual obliga a aumentar el tamaño de la muestra por encima de lo estadísticamente requerido para darle más apariencia de representatividad. En ambos casos, debido a que no todo el mundo es igual, se cuestiona el uso del muestreo en la forma convencional.

\subsubsection{La medición}

Bajo los nuevos supuestos heurísticos, no es sostenible el principio de dar a todos los investigados el mismo estímulo, que está en la base de la posibilidad de realizar encuestas. Si se parte de que es imposible ser neutro sin dejar de ser (ruptura del supuesto de neutralidad del método) y que no existe un valor verdadero que está esperando a ser capturado por el investigador (ruptura del principio de realismo del objeto), entonces no se puede utilizar un referente de verdad para determinar la calidad de lo medido.

Y si no hay alguien con capacidad de conocer la Verdad, dado que todo lo que hacen los sujetos, cualquiera que sea su condición (lego o experto) es subjetivo ${ }^{7}$, ¿por qué delegar en los científicos (los expertos) para que decidan por el resto de la población? En estas nuevas circunstancias, se hace necesario pensar en una investigación participativa, donde se fundan los antiguos objeto y sujeto de la investigación, ahora solo parcialmente escindidos, y donde el criterio para ver la bondad (que no "calidad», que es un término mucho más "técnico») de la investigación remita no a la reproducción (imposible) de lo observado, sino a su construcción, negociada socialmente y, por ende, aceptada por la población.

\subsubsection{El análisis}

A nivel descriptivo, la principal diferencia con el uso convencional de la encuesta, basada en los supuestos también convencionales, estribaría en desarrollar análisis que permitieran explicar la homogeneidad que se observa en la sociedad, para lo cual, tal como hemos explicado, el análisis a partir de una matriz de datos que solo contiene variables, o que solo explica variabilidad, resulta inapropiado. ¿Es posible pensar en una estadística construida sobre alguna medida de explicación de la homogeneidad, a la manera como la estadística actual se construye sobre la explicación de la variabilidad? ¿¿ una estadística que tenga en cuenta la presencia, además de variables, de constantes?

Por lo demás, si decimos que los individuos no son iguales, ¿̨cómo vamos a combinar sus opiniones, dándoles a todas igual peso, para producir una opinión media o calcular cualquier otra variable estadística? ¿Qué valor podría tener esta variable estadística como herramienta predictiva? ¿Y qué valor tiene en este sentido hablar de la opinión pública, medida precisamente mediante

7. Jesús Ibáñez, afamado sociólogo ya fallecido, decía que en la vida solo se puede ser objetivo..., para la caza. 
encuestas que utilizan esas variables estadísticas, basadas en ese supuesto? En esta perspectiva, se explicaría que una hipotética encuesta realizada en la Rusia zarista días antes de la Revolución de 1905 habría sido incapaz de preverla, precisamente por hacer el supuesto de igualdad de todas las opiniones individuales y descartando así el papel de individuos o grupos (de presión, se podría decir) singulares, que fueron los que canalizaron las revueltas, y cuyas opiniones, al combinarlas con las del resto, quedarían diluidas.

A nivel inferencial, la falta de un valor verdadero impide calcular los errores fijos y variables que se utilizan para estimar los parámetros poblacionales a partir de los valores estadísticos obtenidos en una muestra. Esta circunstancia invalidaría el uso convencional del muestreo, cuya justificación precisamente es medir (que no evitar) los errores que se producen con su utilización.

\subsection{Cambios en el ámbito de la sociedad}

Si la estadística - base fundamental de la encuesta - es la ciencia del estado moderno de corte occidental (statuum scientia), el cambio de este tipo de forma política es la condición necesaria tanto para desarrollar otro tipo de estadística (herramienta cognitiva), como para su negación, según cual sea el alcance (menor o mayor, respectivamente) de dicho cambio y la eventual sustitución por otra herramienta cognitiva distinta.

En el nivel de los supuestos heurísticos, habría que sustituir el uso de la razón por la negociación. Dado que no hay UNA verdad a la que acceder a través de la razón, se deja vía libre a la expresión de los intereses de las personas, tan legítimos - y, por lo tanto, sin que sea necesario ocultarlos- como la cantidad de verdad (razón) sobre la que se sustentan. En este sentido, el más importante de todos los cambios sociales sería el que permitiera la negociación entre las personas sin necesidad de apelar a UNA verdad última, de carácter excluyente y sobrenatural; una verdad que, en la actualidad, detentan los expertos, en la medida que dominan el método de llegar a ella (la ciencia, en tanto que la máxima expresión de la razón), como en el pasado la detentaron los sacerdotes, por su capacidad para interpretar las Sagradas Escrituras, que era el método utilizado en su época para acceder a la Verdad.

En los términos que utiliza Richard Rorty al hablar de la ciencia como solidaridad, se trataría de sustituir la búsqueda de la verdad por una «indagación (en cualquier ámbito de la cultura) [que permitiera] lograr una mezcla adecuada de acuerdo no forzoso con desacuerdo tolerante (donde lo que se considera adecuado se determina en esta esfera por ensayo y error)» (Rorty, 1996: 65). La negociación necesaria para conseguir el acuerdo no forzoso habría que plantearla teniendo en cuenta la situación (los intereses) de las personas y no solo su grado de posesión de la Verdad. En términos sociales, esta forma de proceder permitiría el relativismo que hay en la idea de constructivismo social, que, como he dicho, sería el supuesto sobre el que se sustentaría un nuevo tipo de estadística o encuesta (herramienta cognitiva). Dentro de este modelo, habría que concebir la ciencia como conocimiento 
científico, compuesto de razones y de intereses (Solís, 1994), y no sólo de razones, tal como ahora se hace.

En el nivel de los supuestos sociopolíticos, el cambio más importante se derivaría de una sociedad que partiera de la desigualdad de los individuos, no para eliminarla —en nombre de la Igualdad—, sino para respetarla. Ser diferente, pero no superior (si se admite el supuesto anterior, no hay una Verdad de cuya posesión - en mayor o menor grado- se derive un mayor estatus), implica necesariamente la negociación y no la imposición. La experiencia histórica muestra que, después de un par de siglos de buscar la igualdad de las gentes, el fracaso ha sido total: la igualdad se ha trastocado en homogeneidad y, por el contrario, probablemente nos encontremos en el momento de máxima desigualdad en la historia de la humanidad.

Se trataría, pues, de ir más allá de planteamientos del tipo del igualitarismo liberal (Rawls, 1973), formulados desde la necesidad de conjugar igualdades y desigualdades, pero siempre teniendo como norte la igualdad ilustrada. Estos nuevos planteamientos mantendrían la disyunción pero modificando el objetivo, que ahora debe ser respetar la desigualdad.

\section{Conclusión}

A lo largo de las páginas precedentes, hemos tratado de mostrar la necesidad de considerar la encuesta como una herramienta cognitiva, imposible de ser comprendida si no es mirando a sus dos partes constitutivas: una visible, los procedimientos estadísticos que todos identificamos con la encuesta - la parte visible del iceberg, en el símil con este elemento de la naturaleza-, y otra oculta, relacionada con todos los supuestos que hay que hacer para mantenerla operativa - la parte oculta del iceberg, siguiendo con el mismo símil. A su vez, la existencia de los supuestos subyacentes al uso de la encuesta hay que enmarcarlos en los grandes principios informadores de nuestro orden social, sin los cuales carecerían de sentido.

Solo una visión comprensiva de la encuesta puede permitir entender los problemas que surgen en su implementación, en particular los que no son de naturaleza técnica y que, por lo tanto, resulta imposible resolver sin considerar los supuestos que se ven implicados en la solución. Como ejemplificamos en el texto con el problema de la no respuesta y una eventual solución que pasase por pagar a los entrevistados, podemos conseguir que éstos contesten, ahora «asalariados» del investigador, con lo que eliminamos este problema..., pero a costa de introducir otro nuevo relacionado con la medición — ya se sabe que el que paga, manda, y obtiene las respuestas que desea.

En el límite, todas las herramientas cognitivas (los saberes) son productos de una época histórica, dentro de la cual tienen (y a la cual le dan) sentido. En una retroalimentación continua, saberes y épocas interactúan para ir evolucionando en un imperfecto proceso simbiótico. Entiendo que estamos en un momento en el que, vistos los problemas que la rodean, ya no basta con obviar la naturaleza histórica de la encuesta, con lo cual se fuerzan cada vez 
más las maquinarias técnica y estadística para resolverlos (proliferación actual de procedimientos técnicos y estadísticos cada vez más sofisticados) y se hace necesario poner en primer plano reflexiones como las que hacemos en este trabajo. Valga ésta como nuestra contribución, siquiera sea muy básica en algunos aspectos, en esta línea.

\section{Referencias bibliográficas}

ARButhNotT, John (1710). «An argument for the Divine Providence, taken from the constant regularity observed in the Birth of both sexes». Phil. Trans., 27. Reproducción en: Kendall, Maurice y PlacketT, R.L. (eds.) (1977). Studies in the History of Statistics and Probability, II. Londres: Charles Griffin and Company.

Blumer, Herbert (1948). «Public Opinion and Public Opinion Polling». American Sociological Review, 13, 542-549.

Bourdieu, Pierre (1973). «L'opinion publique n'existe pas». Les Temps Modernes, 318, 1292-1309.

- (1984). Questions de sociologie. París: Minuit.

- (2001). Science de la science et reflexivité. París: Raisons d'Agir.

BRYCE, James (1989). The American Commonwealth, 1889. Nueva edición en Indianapolis: Liberty Fund, 1995.

Carmines, Edward G.; Zeller, Richard A. (1979). Reliability and Validity Assessment. Londres: Sage University Paper.

Cea D'Ancona, Ma Ángeles (2005). «La senda tortuosa de la "calidad" de la encuesta». Revista Española de Investigaciones Sociológicas, 111, 75-103.

Cicourel, Aaron (1964). Method and Measurement in Sociology. Nueva York: Free Press.

Converse, Jean M.; Presser, Stanley (1986). Survey Questions: Handcrafting the Standardized Questionnaire. Londres: Sage Publications.

COUMET, Ernest (2000). «La teoría del azar, ¿nació por azar?». Empiria, 3, 209-241.

DERRIDA, Jacques (1992). El otro cabo: La democracia, para otro día. Barcelona: Ediciones del Serbal.

DESROSIÉRES, Alain (1985). «Histoire des formes: statistiques et sciences sociales avant 1940». Revue Française de Sociologie, abril-junio, XXVI-2, 277-310.

- (1988). «Masses, individus, moyénnes: la statistique sociales au XLXéme siécle». Kermes, 2, 41-66.

- (1990). «Cómo fabricar cosas que se sostienen entre sí?: Las ciencias sociales, la estadística y el estado» (traducción mimeografiada de Javier Izquierdo). En: Wagner, WitTrock y Whitíey (eds.). Discourses on Society: The Shaping of the Social Science Disciplines. Sociology of the Science Yearbook, XV, Dordrech: Kluwer, 195-218.

- (1993). La politique des grands nombres. París: La Decouverte.

- (1996). «Reflejar o instituir: la invención de los indicadores estadísticos». Methodologica, 4, 41-57.

Díaz DE RADA, Vidal (2000). Problemas originados por la no respuesta en investigación social: Definición, control y tratamiento. Pamplona: Universidad Pública de Navarra.

FOWLER, Floyd J. y MANGIONI, Thomas W. (1990). Standardized Survey Interviewing: Minimizing Interviewer-Related Error. Londres: Sage Publicactions.

Gallup, George H. y REA, Saul F. (1940). The pulse of democracy: The public-opinion poll and how it works. Nueva York: Simon and Schuster. 
Galton, Francis (1889). Natural Inheritance. Londres: McMillan. Edición parcial de sus obras en castellano: Álvarez PeLÁEZ, Raquel (1988). Francis Galton: Herencia y eugenesia. Madrid: Alianza Universidad.

Goyder, J. (1987). The Silent Minority: Nonrespondents on Sample Surveys. Cambridge: Polity Press.

Goyder, J. y LeIPer, J.M. (1985). «The decline in survey response: A social values interpretation». Sociology, 19, 55-71.

Graunt, John (1661). Observations upon the Bills of Mortality. Edición de Hull, Charles Henry (ed.) (1964). The Economics Writtings of Sir William Petty: Together with the Observations upon the Bills of Mortality, more probably by Captain John Graunt. Nueva York: August M. Kelley Bookseller.

Groves, Robert M. (1987). «Research on Survey Data Quality». Public Opinion Quarterly, 50th aniversario, invierno, S156-S172.

- (1989). Survey Errors and Survey Costs. Nueva York: John Wiley and Sons.

Groves, R.M. y COUPER, M.P. (1998). Nonresponse en Household Interview Surveys. Nueva York: Wiley.

HaBermas, Jurgen (2004). Historia y crítica de la opinión pública. Barcelona: Gustavo Gili.

Hecht, J. (1987). «L'idée de dénombrement jusqu’à la Révolution». En: INSEE: Pour une histoire de la statistique. París: INSEE.

IBÁÑEZ, Jesús (1992). «Perspectivas de la investigación social: el diseño en las tres perspectivas». En: GARCÍA FERRANDO, Manuel; IBÁÑEZ, Jesús y Alvira, Francisco. El análisis de la realidad social: Métodos y técnicas de investigación. Madrid: Alianza Universidad Textos.

Iranzo, J. Manuel; Blanco, J. Rubén; GonzÁlez de la Fe, Teresa; Torres, Cristóbal y Cotillo, Alberto (1995). Sociología de la ciencia y la tecnología. Madrid: Consejo Superior de Investigaciones Científicas.

IRANZO, J. Manuel y BlanCO, J. Rubén (1999). Sociología del conocimiento científico. Madrid: CIS.

KaLTON, Graham (1983). Introduction to Survey Sampling. Londres: Sage University Paper.

KisH, Leslie (1965). Survey Sampling. Nueva York: Wiley.

ORTí, Alfonso (1992). "La apertura y el enfoque cualitativo o estructural: la entrevista abierta semidirectiva y la discusión de grupo». En: GARCÍA FERrANDO, Manuel; IBÁNEZZ, Jesús y AlvirA, Francisco. El análisis de la realidad social: Métodos y técnicas de investigación. Madrid: Alianza Universidad Textos.

PEARSON, Karl (1892). The Grammar of Science. Londres: Scott. Reedición de 1957 sobre la tercera edición de Pearson, de 1911. Nueva York: Meridian Books, Inc.

PetTy, William (1676). Political Arithmetic. Edición de Charles Henry Hull (1964). The Economics Writtings of Sir William Petty: Together with the Observations upon the Bills of Mortality, more probably by Captain John Graunt. Nueva York: August M. Kelley Bookseller.

PORTER, Theodore (1985). The Rise of Statistical Thinking. New Jersey: Princenton University Press.

Presser, S.; Couper, M.P.; Lessler, J.T.; Martin, E.; Martin, J.; Rothgeb, J.M. y Singer, E. (2004a). «Methods for testing and evaluating survey questions». Public Opinion Quarterly, 68 (1), 109-130.

Presser, S.; Rothgeb, J.; Couper, M.; Lessler, J.; Martin, E.; Martin, J. y Singer, E. (eds.) (2004b). Questionnaire Development Evaluation and Testing Methods. Nueva York: Wiley. 
QueTELET, Adolphe (1835). Sur l'homme et le développent de ses facultés, ou Essai de physique sociale. París: Bachelier, Imprimeur-Libraire.

- (1869). Physique sociale ou Essai sur le développent des facultés de l'homme. Bruselas: C. Musquardt, Libbreur-Editeur. Reedición anotada: VILQUIN, Eric y SANDERSON, Jean Paul (1997). Bruselas: Academie Royale de Belgique.

RaWls, John (1973). Teoría de la justicia. México: Fondo de Cultura Económica.

RORTY, Richard (1996). Objetividad, relativismo y verdad: Escritos filosóficos. Barcelona: Paidós.

SÁnChez CARrión, Juan Javier (1996). «La calidad de la encuesta». Papers, 48, 127 146.

- (1999a). Manual de análisis estadistico de los datos. Madrid: Alianza Editorial.

- (1999b). «Quetelet y la sociología». Revista Española de Investigaciones Sociológicas, 87, 291-322.

- (2000a). «Sociología, orden social y modelización estadística: Quetelet y el hombre medio». Empiria, 3, 49-71.

- (2000b). La bondad de la encuesta: el caso de la no respuesta. Madrid: Alianza Editorial Materiales.

- (2001a). «Estadística, orden natural y orden social». Papers, 63/64, 33-46.

- (2001b). «La encuesta, el iceberg y el estado». Inguriak, 29, 21-30.

- (2001c). «Reflexiones sociológicas sobre el recuento de la población (a partir del siglo XVII). Historia y Política, 3, 137-159.

SARIS, Willem E.; VAN DER VELD, William y GallHOFER, Irmtraud (2004). «Development improvements of questionnaires using predictions of realibily and validity». En: Presser, S.; Rothgeb, J.; Couper, M.; Lessler, J.; Martin, E.; Martin, J. y Singer, E. (eds.). Questionnaire Development Evaluation and Testing Methods. Nueva York: Wiley.

SARIS, W.E. y Gallhofer, I. (2007). Design, Evaluation, and Analysis of Questionnaires for Survey Research. Nueva York: Wiley.

SCHUMAN, Howard y Presser, Stanley (1981). Questions and answers in Attitude Surveys: Experiments on Questions Form, Wording and Context. San Diego (CA): Academic Press.

Solís, Carlos (1994). Razones e intereses: La historia de la ciencia después de Kuhn. Barcelona: Paidós.

STOOP, I.A. (2006). The hunt for the last respondent: Nonresponse in sample surveys. The Hague: Social and Cultural Planning Office of the Netherlands.

Stoop, I.A.; Billiet, J.; Koch, A. y Fitzgerald, R. (2010). Survey Nonresponse in Europe. Nueva York: Wiley.

Stouffer, Samuel A. (1949). The American Soldier. Princeton: Princeton University Press.

Torres Amat, Félix (1894). La Sagrada Biblia. Antiguo Testamento. Barcelona: Imprenta y Librería de los Hnos. Subirana.

TukEY, John Wilder (1977). Exploratory Data Analysis. Londres: Addison-Wesley. 